\title{
Alterations in regional homogeneity of resting-state brain activity in internet gaming addicts
}

Guangheng Dong ${ }^{1 *}$, Jie Huang ${ }^{1}$ and Xiaoxia Du²

\begin{abstract}
Backgrounds: Internet gaming addiction (IGA), as a subtype of internet addiction disorder, is rapidly becoming a prevalent mental health concern around the world. The neurobiological underpinnings of IGA should be studied to unravel the potential heterogeneity of IGA. This study investigated the brain functions in IGA patients with resting-state fMRI.

Methods: Fifteen IGA subjects and fourteen healthy controls participated in this study. Regional homogeneity (ReHo) measures were used to detect the abnormal functional integrations.

Results: Comparing to the healthy controls, IGA subjects show enhanced ReHo in brainstem, inferior parietal lobule, left posterior cerebellum, and left middle frontal gyrus. All of these regions are thought related with sensory-motor coordination. In addition, IGA subjects show decreased ReHo in temporal, occipital and parietal brain regions. These regions are thought responsible for visual and auditory functions.

Conclusions: Our results suggest that long-time online game playing enhanced the brain synchronization in sensory-motor coordination related brain regions and decreased the excitability in visual and auditory related brain regions.
\end{abstract}

Keywords: Internet addiction disorder, Internet gaming addiction, Resting-state fMRI

\section{Background}

Internet addiction disorder (IAD) is usually defined as the inability of an individual to control his or her use of the internet. This inability will eventually cause psychological, social, and/or work difficulties [1-4]. As the world's fastest growing 'addiction', IAD has been considered as a serious public health issue [1,5,6]. Although significant prevalence estimations and associations with adverse consequences have been addressed [1,7-11], few studies focused on the neurobiological underpinnings of IAD [12-14]. Thus, investigations on the neurobiological basis of IAD are necessary and important. Discovery of the biomarkers of IAD will provide valuable information on unraveling the underpinnings of IAD and on assessing proper treatment strategies.

\footnotetext{
* Correspondence: dongguangheng@zjnu.edu.cn

'Department of Psychology, Zhejiang Normal University, 688 of Yingbin

Road, Jinhua city, Zhejiang Province, P.R.China

Full list of author information is available at the end of the article
}

IAD is also considered as a behavioral addiction and may share similar neuropsychological (i.e., development of euphoria, craving, and tolerance) and personality characteristics with other addictions [15], especially behavioral addiction, for example, gambling addiction [16]. Internet addiction consists at least three subtypes: Internet gaming addiction (IGA), sexual preoccupations, and email/text messaging [12,14]. Comparing to other subtypes of IAD, internet gaming addiction, the most important subtype of IAD, exhibits some specific features such as role-playing in the virtual world. Few studies have addressed the brain functional changes in people who are addicted to the internet games. Previous studies showed that mental disorders could change the spontaneous activity in the brain [17-19]. Researchers believed that internet addiction is an impulse disorder or is at least related to it [20]. Thus, IGA may share similar neuropsychological and personality characteristics with other mental disorders or behavioral addictions $[15,16]$. 
Potential changes in brain are expected in IGA patients by analogy with other mental disorders.

Resting-state functional MRI has been increasingly used to investigate the integration of neural activities at a resting state when no task is performed [21]. Although resting-state fMRI is a relatively new technique, many exciting findings have been reported in the past several years (for a review see [22]). Nowadays, this technique has been successfully used to detect abnormal functional integration in some mental disorders [23,24]. Regional homogeneity (ReHo) is a widely used method in restingstate fMRI studies $[21,25,26]$. ReHo measures the functional coherence of a given voxel with its nearest neighbors, which can be used to evaluate resting-state brain activities based on the hypothesis that significant brain activities would more likely occur in clusters than in a single voxel [27]. ReHo index could be regarded as a measurement for investigating human brain activities in the resting state and may be useful for revealing the complexity of human brain function $[28,29]$.

Online game playing requires players staring at the computer screen and enduring the sound of the game for a long time. Previous studies have shown that noise could cause hearing problem [30-33], and long-time hypertension visual attention could cause serious visual acuity decrease or vision loss [34]. Thus, the long-time game playing might impair the visual or hearing functions and these changes could be detected in relevant brain regions. In this study, we hypothesized that longtime online game playing changed the brain functions in the regions that are related with vision or hearing. To address this issue, we employed resting-state fMRI to examine the spontaneous fluctuations of brain activities in IGA subjects in this study.

\section{Methods and procedure Subject}

We examined a group of 15 (16-1) male IAD participants (i.e., male, right handed, non-smokers) aged $24.2 \pm 3.5$ years (mean \pm SD) and a group of $14(15-1)$ age matched, healthy males aged $24.6 \pm 3.8$ years (mean \pm $\mathrm{SD})$ (i.e., right handed, non-smokers). Our study focused on male subjects because the prevalence of IAD in men is much higher than that in women [35]. IAD subjects were recruited through advertisements. All participants underwent structured psychiatric interviews (M.I.N.I.) performed by an experienced psychiatrist with an administration time of approximately 15 minutes. The MINI was designed to meet the need for a short but accurate structured psychiatric interview for multicenter clinical trials and epidemiology studies [36]. All participants were free of Axis I psychiatric disorders listed in M.I.N.I. Depression was further assessed using the Beck
Depression Inventory [37]. Anyone who scored more than 5 would be excluded from our study.

All IAD subjects had a diagnosis of Young's online internet addiction test [38], which consists of 20 items associated with online internet use including psychological dependence, compulsive use, and withdrawal, as well as related problems in school or work, sleep, family, and time management. For each item, a graded response is selected from $1=$ "Rarely" to $5=$ "Always", or "Does not Apply". The IAT is proved to be a valid and reliable instrument that can be used in classifying IAD $[39,40]$. People who scored more than 50 were considered to experience occasional or frequent problems because of the internet. Those who scored more than 80 were considered to have significant problems in their lives [38]. In the present study, the threshold cut-off we used is 80 in the internet addiction test. Estimates of the size of the group of 'addicted gamers' are defined by applying various cut-off points to scales measuring symptoms of internet addiction [41,42]. In present study, we added some specific limitations on the established measures of internet addiction, such as 'you spend most of your time playing online games $(>80 \%)$ '.

The controls were also measured with the same process. Controls scored lower than 20 in Young's test $(16.3 \pm 4.3$ mean $\pm \mathrm{SD})$. All participants were medication free and were instructed not to use any substance of abuse, including coffee, on the day of the scan. No participant had previous experience with cocaine or marijuana. The human investigation committee of Zhejiang normal university approved this research. All subjects signed a written informed consent.

\section{Scanning process}

The 'resting state' was defined as no specific cognitive task during the fMRI scan. Participants were required simply to keep still, close their eyes and not to think of anything systematically $[27,28]$. Magnetic resonance imaging scanning MRI data were acquired using a Siemens Trio $3 \mathrm{~T}$ scanner (Siemens, Erlangen, Germany) in EastChina Normal University. Participants lay supine with head snugly fixed by belt and foam pads to minimize head movement. The resting-state functional images were acquired by using an echo-planar imaging sequence with the following parameters: interleaved, 33 axial slices, thickness $=3.0 \mathrm{~mm}$, in-plane resolution $=64^{*}$ 64, repetition time $=2000 \mathrm{~ms}$, echo time $=30 \mathrm{~ms}$, flip angle $=90$, field of view $=220 * 220 \mathrm{~mm}, 240$ volumes (8 min). Structural images were collected using a T1weighted three-dimensional spoiled gradient-recalled sequence was acquired covering the whole brain (176 slices, repetition time $=1700 \mathrm{~ms}$, echo time $\mathrm{TE}=3.93 \mathrm{~ms}$, slice thickness $=1.0 \mathrm{~mm}$, skip $=0 \mathrm{~mm}$, flip angle $=15$, inversion 
time $1100 \mathrm{~ms}$, field of view $=240 * 240 \mathrm{~mm}$, in-plane resolution $=256^{*} 256$ ).

\section{Preprocessing of image data}

Preprocessing and ReHo process were using the software DPARSF (Data Processing Assistant for Resting-State fMRI. www.restfmri.net), a MATLAB toolbox for 'pipeline' data analysis of resting-state fMRI $[43,44]$. DPARSF is based on some functions in SPM and REST. The main pre-processing steps and parameters are: The first 10 volumes of each functional time series were discarded for participant adaptation to the scanning. Then, the image preprocessing, including slice timing, head motion correction and spatial normalization were conducted. After the procedure of head motion correction, the values for translation $(\mathrm{mm})$ and rotation (degrees) were obtained at each time point in six parameters (three for translation and three for rotation) for each participant. Participants ( 1 from IAD group and 2 from control group) with head motion more than $2.0 \mathrm{~mm}$ maximum displacement in any direction of $\mathrm{x}, \mathrm{y}$, and $\mathrm{z}$ or $1^{\circ}$ of any angular motion throughout the course of scan were excluded from further analysis. The fMRI data were temporally band-pass filtered $(0.01-0.08 \mathrm{~Hz})$ to reduce the low-frequency drift and physiological high-frequency respiratory and cardiac noise. Then the time-series of images of each subject were motion-corrected using a least squares approach and a six-parameter (rigid body) linear transformation. Individual ReHo map was generated by calculating Kendall's coefficient concordance (with value from 0 to 1 ) of time series of a given voxel with those of its nearest neighbors (26 voxels) in a voxel-wise way. Spatial smoothing was conducted on the ReHo maps with a Gaussian kernel of $4 * 4 * 4 \mathrm{~mm}^{3}$ full-width at half-maximum. The intracranial voxels were extracted to make a mask [43]. For standardization purposes, each individual ReHo map was divided by its own mean ReHo within the mask.

\section{Statistical analyses \\ ReHo analysis}

Two-sample t-test was performed on the normalized individual ReHo maps. The resultant statistical map was set at a combined threshold of $p_{F D R}<0.05$ and a minimum cluster size is 10 voxels $\left(270 \mathrm{~mm}^{3}\right)$.

\section{Correlation analysis}

We calculated the correlation between IAT scores and the averaged peak values in all these survived clusters after correction in brainstem, inferior parietal lobule, left posterior cerebellum, and left middle frontal gyrus in IAD subjects. In addition, we also calculated the correlation between IAT score and the averaged peak values in all these survived clusters after correction in temporal, occipital and parietal brain regions in IAD subject.

\section{Functional connectivity analysis}

The inferior parietal lobule of the left hemisphere lies at the junction of the auditory, visual, and somatosensory cortexes. Thus, we selected this region as the seed region. We calculated the functional connectivity between the seed and other brain regions in the ANOVA analysis showing significant ReHo difference between the two groups (IAD-Controls).

\section{Results}

ReHo results

As shows in Table 1 and Figure 1, the IAD subjects displayed significantly increased ReHo in brainstem (left and right), inferior parietal lobule (left and right), left

Table 1 Brain areas of ReHo difference between two groups (IAD - Controls)

\begin{tabular}{|c|c|c|c|c|}
\hline Main areas & Hemisphere & Peak MNI coordinates $(x, y, z)$ & Voxels & Peak $t$ value \\
\hline \multicolumn{5}{|l|}{ Increased ReHo in IAD } \\
\hline Right Brainstem & R & $3,-6,-15$ & 23 & 5.0501 \\
\hline Left Brainstem & L & $-3,-21,-12$ & 16 & 3.6839 \\
\hline Inferior Parietal Lobule & R & $63,-30,33$ & 19 & 3.5916 \\
\hline Inferior Parietal Lobule & L & $-60,-36,39$ & 18 & 4.3319 \\
\hline Cerebellum Posterior Lobe & L & $-48,-33,-51$ & 47 & 4.7067 \\
\hline Middle Frontal Gyrus & L & $-60,27,30$ & 63 & 4.3593 \\
\hline \multicolumn{5}{|l|}{ Decreased ReHo in IAD } \\
\hline Inferior Temporal Gyrus & L & $-54,-42,-24$ & 19 & -4.3287 \\
\hline Superior Temporal Gyrus & L & $-39,33-33$ & 25 & -5.2271 \\
\hline Occipital Lobe, limbic lobe & L & $-24,-63,3$ & 20 & -3.9607 \\
\hline Parietal Lobe (Postcentral) & L & $-63,-6,15$ & 19 & -3.8942 \\
\hline Middle Cingulate Gyrus & L & $0,-15,39$ & 23 & -4.9526 \\
\hline
\end{tabular}

Voxel size $=3 * 3 * 3$ mm. $p<0.05$ FDR corrected and at least 10 voxels. 


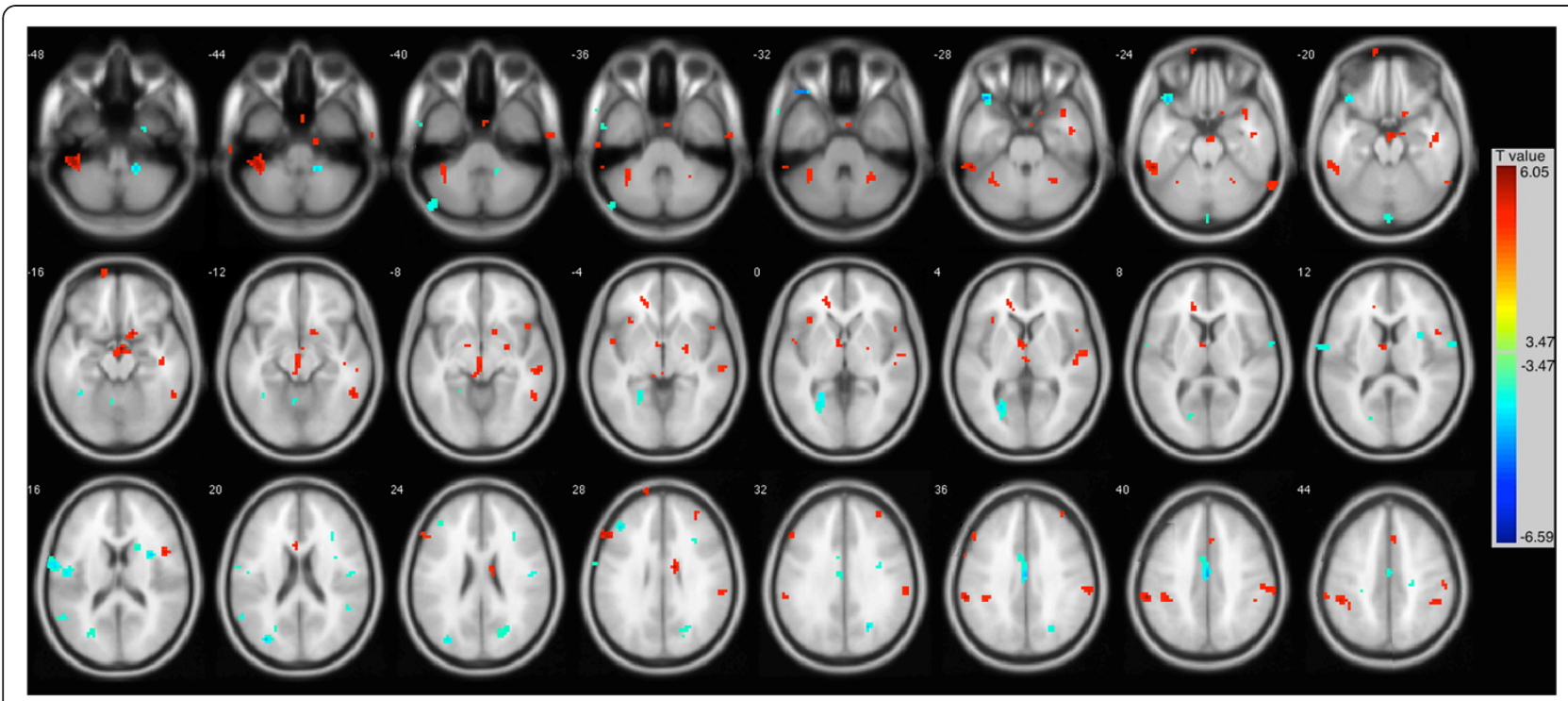

Figure 1 Increased and decreased ReHo in IAD when comparing to healthy controls. Voxel size $=3^{*} 3^{*} 3 . p<0.05$ FDR corrected.

posterior cerebellum, and left middle frontal gyrus when comparing with healthy controls. In addition, significantly decreased ReHo was discovered in left superior and inferior temporal gyrus, left occipital lobe and parietal lobe.

\section{Correlation results}

The correlation between IAT scores and the averaged peak values in all these survived clusters after correction in brainstem, inferior parietal lobule, left posterior cerebellum, and left middle frontal gyrus in IAD subjects is 0.080 . The correlation between IAT score and the averaged peak values in all these survived clusters after correction in temporal, occipital and parietal brain regions in IAD subject is 0.205 . Maybe because there are only 15 IAD subjects in present study, no significant correlation was found among these factors (Figure 2).

\section{Functional connectivity results}

Table 2 shows the functional connectivity results. Although no significant difference was found between the IAD group and healthy controls, but the results still can provide some support to our speculations in this study (Table 2).

\section{Discussion}

Decreased or increased ReHo in brain suggest neural functions in certain regions are less or more synchronized [27]. In this study, ReHo analysis depicted several brain areas, where local BOLD signal coherence are different in IGA subjects when comparing with that of healthy controls.
Enhanced sensory-motor coordinate ability

Enhanced ReHo was found in brainstem, inferior parietal lobule, left posterior cerebellum, and left middle frontal gyrus. All these areas are responsible for coordinating motor-sensory ability to some degrees. The brainstem serves as a conduit for many ascending and descending pathways. It is an extremely important part of the brain as the nerve connections of the motor and sensory systems from brain to the rest of the body $[45,46]$. The cerebellum is a region of the brain that plays an important role in motor control [47]. The cerebellum does not initiate movement, it receives input from sensory systems and from other parts of the brain, and integrates these inputs to fine tune motor activity [48]. The inferior parietal lobule of the left hemisphere lies at the junction of the auditory, visual, and somatosensory cortexes, with which it is massively connected. In addition, the neurons in this lobule can process different kinds of stimuli (auditory, visual, sensorimotor, etc.) simultaneously $[49,50]$. Functional dissociation between the left and right middle frontal gyrus has been suggested in working memory, which is strongly related to the involved mental operations [51].

Considering the characteristics of IAD and the functions of the brain regions we discussed above, we may infer that IAD subjects have enhanced coordinate ability in motor and sensory systems. When playing online game, players need to build avatars in their virtual world [52]. They also need to compete or cooperate with other players to fight against some enemies. Their actions, such as fighting, killing, shooting, using all kinds weapons and so on, are complicated and challenging. Players need to control their avatars skillfully to accomplish 

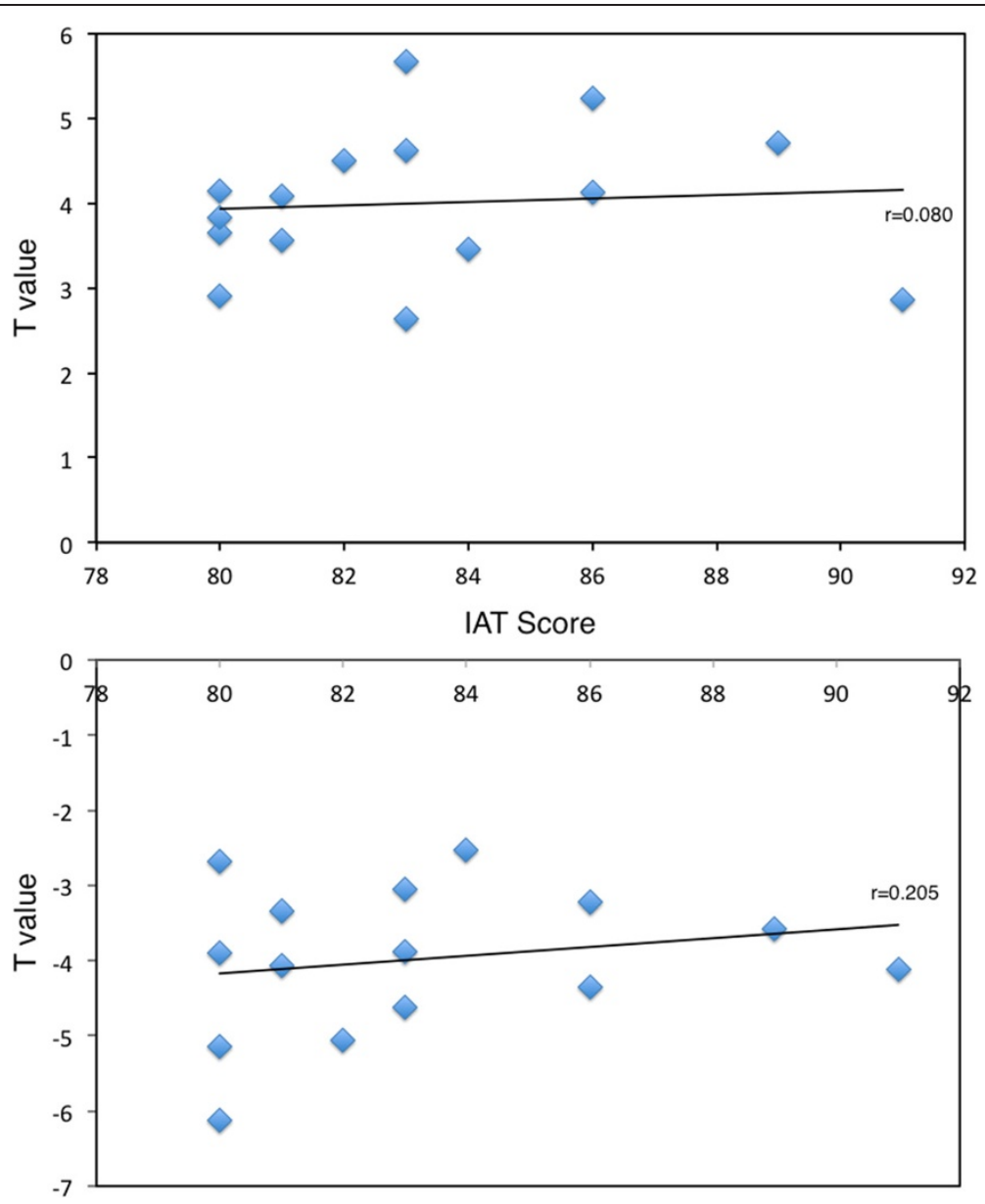

Figure 2 Correlation between the mean peak values survived after correction (hyper-activation) and IAT scores (upper); Correlation between the mean peak values survived after correction (hypo-activation) and IAT scores (upper) (bottom).

their tasks. Thus, the online game playing requires players to coordinate several systems, including the sensory system, motor control, motor coordinate and information processing system. In addition, online internet games can not be completed in a set time because of the regular introduction of new contents, which require players to continue playing to 'keep up' with the game [53]. These games may be addictive because they are particularly good at inducing operant conditioning via variable-ratio reinforcement schedules (a highly effective

Table 2 Differences of functional connectivity in the brain regions survived in ReHo analysis between IAD and HC (Seed: inferior parietal lobule in the left hemisphere)

\begin{tabular}{lllll}
\hline Location & Hemisphere & Peak MNI coordinates $(\mathbf{x}, \mathbf{y}, \mathbf{z})$ & T value (IAD-Control) & P value \\
\hline Right Brainstem & $\mathrm{R}$ & $3,-6,-15$ & 3.13 & $p>0.05$ \\
Left Brainstem & $\mathrm{L}$ & $-3,-21,-12$ & 2.37 & $\mathbf{p}$ \\
Inferior Parietal Lobule & $\mathrm{R}$ & $63,-30,33$ & 3.96 & $p>05$ \\
Cerebellum Posterior Lobe & $\mathrm{L}$ & $-48,-33,-51$ & 1.71 & $p>05$ \\
Middle Frontal Gyrus & $\mathrm{L}$ & $-60,27,30$ & 0.89 & $p .05$ \\
Inferior Temporal Gyrus & $\mathrm{L}$ & $-54,-42,-24$ & 1.38 & $p>0.05$ \\
Superior Temporal Gyrus & $\mathrm{L}$ & $-39,33-33$ & 1.84 & $p>0.05$ \\
Occipital Lobe, limbic lobe & $\mathrm{L}$ & $-24,-63,3$ & 0.69 & $p>0.05$ \\
Parietal Lobe (Postcentral) & $\mathrm{L}$ & $-63,-6,15$ & 2.94 & $p>0.05$ \\
Middle Cingulate Gyrus & $\mathrm{L}$ & $0,-15,39$ & 1.52 & $p>0.05$ \\
\hline
\end{tabular}


conditioning paradigm [54]). From what we discussed above, we may infer that the long-term game playing enhanced coordinate ability among motor, sensory and information processing systems in brain.

\section{Impaired visual and auditory function}

Consistent with our hypothesis, the IAD subjects show decreased ReHo in temporal, occipital and parietal brain regions when comparing with the healthy controls. These regions are thought to be responsible for visual and auditory functions. The superior temporal gyrus contains several important structures of the brain, including the location of the primary auditory cortex, which is responsible for the sensation of sound [55]. Inferior temporal gyrus is one of the higher levels of the ventral stream of audio and visual processing, associated with the representation of complex object features $[56,57]$. The occipital lobe is the visual processing center of the mammalian brain containing most of the anatomical region of the visual cortex $[58,59]$. The parietal lobe integrates sensory information from different modalities, particularly determining spatial sense and navigation [60-62].

The decreased ReHo in visual and auditory related brain regions might suggest the decreased synchronization in IGA subjects. Considering the features of IGA, we can infer that this may be the result of longtime game playing. The gaming process requires players to pay full attention to each subtle change in the screen, to endure the noisy sound of the game for a long time (usually more than 10 hours a day). The visual and auditory related brain regions have been stimulated for a long time, which made them hardly to be excited or have a decreased excitability. As we mentioned in the introduction section, long time hypertension of visual attention can impair subjects' visual functions and noise can impair their hearing abilities [17-19]. Thus, we may infer that the long-time game playing (expose to visual and auditory stimuli) may impair player's visual and auditory ability, which could be indexed by the decreased ReHo in this study.

\section{Limitation}

Our results may sound more persuasive if we include a group of video game addicts as another control group. From comparisons between IGA subjects and video game addicts, we may find some specific features of IGA. In addition, if we had vision and hearing tests on the subjects before scan and take these variables as regressors in data analysis, we could get better scientific results. These issues will be emphasized in our future studies. Third, correlation and functional connectivity results cannot provide strong support to the conclusions about the impairment in visual auditory ability. Future studies should involve more participants to investigate this issue.

\section{Conclusions}

From what we discussed above, we can conclude that long-time online game playing enhanced the sensorymotor coordinate ability and impaired participants' visual and auditory functions. All these results can help policy-makers or individuals understand the influence of internet gaming addiction. First, this research can help us understand the mechanism of addiction, find if it shares characteristics with other types of addictions; Second, this research revealed the influence of internet games, especially its negative aspects to our brain mechanism.

\section{Competing interests}

The authors do not have an affiliation with or financial interest in any organization that might pose a competing interest.

\section{Authors' contributions}

GD took part in designing the research and the manuscript preparation. $\mathrm{JH}$ analyzed the data. XD collected the data and took part in manuscript preparation. All authors read and approved the final manuscript.

\section{Acknowledgements}

This research was supported by National Science Foundation of China (30900405). The funder had no role in research design, data collection and analysis, decision to publish, or preparation of the manuscript.

\section{Author details}

${ }^{1}$ Department of Psychology, Zhejiang Normal University, 688 of Yingbin Road, Jinhua city, Zhejiang Province, P.R.China. ${ }^{2}$ Department of Physics, Shanghai Key Laboratory of Magnetic Resonance, East China Normal University, Shanghai, P.R.China.

Received: 23 March 2012 Accepted: 16 August 2012 Published: 18 August 2012

\section{References}

1. Young K: Internet addiction: the emergence of a new clinical disorder. Cyberpsychol Behav 1998, 1(3):237-244.

2. Davis RA: Cognitive-behavioral model of pathological Internet use. Comput Hum Behav 2001, 17(2):187-195.

3. Young KS, Rogers RC: The relationship between depression and internet addiction. Cyberpsychol Behav 1998, 1(1):25-28.

4. Young KS: Internet addiction: the emergence of a new clinical disorder. Cyberpsychol Behav 1998, 1(3):237-244.

5. van den Eijnden RJJM, Spijkerman R, Vermulst AA, van Rooij TJ, Engels RCME: Compulsive Internet Use Among Adolescents: Bidirectional Parent-child Relationships. J Abnorm Child Psychol 2010, 38(1):77-89.

6. Dong G, Lu Q, Zhou H, Zhao X: Precursor or sequela: pathological disorders in people with Internet addiction disorder. PLOS One 2011, 6(2):e14703.

7. Kim JH, Lau CH, Cheuk K-K, Kan P, Hui HLC, Griffiths SM: Predictors of heavy Internet use and associations with health-promoting and health risk behaviors among Hong Kong university students. J Adolesc 2010, 33(1):215-220.

8. Niemz K, Griffiths M, Banyard P: Prevalence of pathological Internet use among university students and correlations with self-esteem, the General Health Questionnaire (GHQ), and disinhibition. Cyberpsychol Behav 2005, 8(6):562-570.

9. Dong G, Huang J, Du X: Enhanced reward sensitivity and decreased loss sensitivity in Internet addicts: an fMRI study during a guessing task. J Psychiatr Res 2011, 45(11):1525-1529. 
10. Dong G, Devito E, Huang J, Du X: Diffusion tensor imaging reveals thalamus and posterior cingulate cortex abnormalities in internet gaming addicts. J Psychiatr Res 2012, 46(9):1212-1216.

11. Dong G, Zhou H, Zhao X: Male Internet addicts show impaired executive control ability: evidence from a color-word Stroop task. Neurosci Lett 2011, 499(2):114-118.

12. Block JJ: Prevalence Underestimated in Problematic Internet Use Study. CNS Spectr 2006, 12:14-15.

13. Liu T, Potenza MN: Problematic Internet Use: Clinical Implications. CNS Spectr 2007, 12(6):453-466.

14. Dong G, Lu Q, Zhou H, Zhao X: Impulse inhibition in people with internet addiction disorder: electrophysiological evidence from a Go/NoGo study. Neurosci Lett 2010, 485(2):138-142.

15. Grant JE, Potenza MN, Weinstein A, Gorelick DA: Introduction to Behavioral Addictions. Am J Drug Alcohol Abuse 2010, 36(5):233-241.

16. Potenza MN, Leung H-C, Blumberg HP, Peterson BS, Fulbright RK, Lacadie CM, Skudlarski P, Gore JC: An fMRI Stroop Task Study of Ventromedial Prefrontal Cortical Function in Pathological Gamblers. Am J Psychiatry 2003, 160(11):1990-1994.

17. Peng DH, Jiang KD, Fang YR, Xu YF, Shen T, Long XY, Liu J, Zang YF: Decreased regional homogeneity in major depression as revealed by resting-state functional magnetic resonance imaging. Chin Med J (Engl) 2011, 124(3):369-373.

18. Paakki JJ, Rahko J, Long X, Moilanen I, Tervonen O, Nikkinen J, Starck T, Remes J, Hurtig T, Haapsamo $\mathrm{H}$, et al: Alterations in regional homogeneity of resting-state brain activity in autism spectrum disorders. Brain Res 2010, 1321:169-179.

19. Wu T, Long X, Zang Y, Wang L, Hallett M, Li K, Chan P: Regional homogeneity changes in patients with Parkinson's disease. Hum Brain Mapp 2009, 30(5):1502-1510.

20. Beard KW, Wolf EM: Modification in the proposed diagnostic criteria for Internet addiction. Cyberpsychol Behav 2001, 4(3):377-383.

21. Liu Y, Wang K, Yu C, He Y, Zhou Y, Liang M, Wang L, Jiang T: Regional homogeneity, functional connectivity and imaging markers of Alzheimer's disease: a review of resting-state fMRI studies. Neuropsychologia 2008, 46(6):1648-1656.

22. Fox MD, Raichle ME: Spontaneous fluctuations in brain activity observed with functional magnetic resonance imaging. Nat Rev Neurosci 2007, 8 (9):700-711.

23. Deco G, Jirsa VK, Mclntosh AR: Emerging concepts for the dynamical organization of resting-state activity in the brain. Nat Rev Neurosci 2011, 12(1):43-56.

24. van den Heuvel MP, Hulshoff Pol HE: Exploring the brain network: a review on resting-state $\mathrm{fMRI}$ functional connectivity. Eur Neuropsychopharmacol 2010, 20(8):519-534.

25. Zhang Z, Liu Y, Jiang T, Zhou B, An N, Dai H, Wang P, Niu Y, Wang L, Zhang $X$ : Altered spontaneous activity in Alzheimer's disease and mild cognitive impairment revealed by Regional Homogeneity. Neuroimage 2011, 59(2):1429-1440.

26. Wang $L$, Song M, Jiang T, Zhang Y, Yu C: Regional homogeneity of the resting-state brain activity correlates with individual intelligence. Neurosci Lett 2011, 488(3):275-278.

27. Zang Y, Jiang T, Lu Y, He Y, Tian L: Regional homogeneity approach to fMRI data analysis. Neuroimage 2004, 22(1):394-400.

28. You H, Wang J, Wang H, Zang YF, Zheng FL, Meng CL, Feng F: Altered regional homogeneity in motor cortices in patients with multiple system atrophy. Neurosci Lett 2011, 502(1):18-23.

29. Wu T, Zang Y, Wang L, Long $X$, Li K, Chan P: Normal aging decreases regional homogeneity of the motor areas in the resting state. Neurosci Lett 2007, 423(3):189-193.

30. Bovo R, Ciorba A, Martini A: Environmental and genetic factors in agerelated hearing impairment. Aging Clin Exp Res 2011, 23(1):3-10.

31. Van Eyken E, Van Camp G, Van Laer L: The complexity of age-related hearing impairment: contributing environmental and genetic factors. Audiol Neurootol 2007, 12(6):345-358.

32. Fransen $E$, Lemkens $N$, Van Laer L, Van Camp G: Age-related hearing impairment (ARHI): environmental risk factors and genetic prospects. Exp Gerontol 2003, 38(4):353-359.

33. Pilati N, Ison MJ, Barker M, Mulheran M, Large CH, Forsythe ID, Matthias J, Hamann M: Mechanisms contributing to central excitability changes during hearing loss. Proc Natl Acad Sci USA 2012, 109(21):8292-8297.
34. DellaCroce JT, Vitale AT: Hypertension and the eye. Curr Opin Ophthalmol 2008, 19(6):493-498.

35. Report on Chinese youth internet addiction disorder. In. Beijing: Chinese Youth association of Internet; 2008:1-17.

36. Lecrubier Y, Sheehan DV, Weiller E, Amorim P, Bonora I, Harnett Sheehan K, Janavs J, Dunbar GC: The Mini International Neuropsychiatric Interview (MINI). A short diagnostic structured interview: reliability and validity according to the CIDI. European Psychiatry 1997, 12(5):224-231.

37. Beck AT, Ward CH, Mendelson M, Mock J, Erbaugh J: An Inventory for Measuring Depression. Arch Gen Psychiatry 1961, 4(6):561-571.

38. Internet Addiction Test (IAT). http://netaddiction.com/.

39. Widyanto L, McMurran M: The psychometric properties of the internet addiction test. Cyberpsychol Behav 2004, 7(4):443-450.

40. Widyanto L, Griffiths MD, Brunsden V: A psychometric comparison of the Internet Addiction Test, the Internet-Related Problem Scale, and self-diagnosis. Cyberpsychol Behav Soc Netw 2011, 14(3):141-149.

41. Gentile D: Pathological Video-Game Use Among Youth Ages 8 to 18: A National Study (vol 20, pg 594, 2009). Psychol Sci 2009, 20(6):785-785

42. Lemmens JS, Valkenburg PM, Peter J: Development and validation of a game addiction scale for Adolescents. Media Psychology 2009, 12(1):77-95

43. Yan C-G, Zang Y-F: DPARSF: A MATLAB Toolbox for "Pipeline" Data Analysis of Resting-State fMRI. Front Syst Neurosci 2010, 4:13.

44. Song XW, Dong ZY, Long XY, Li SF, Zuo XN, Zhu CZ, He Y, Yan CG, Zang YF: REST: A Toolkit for Resting-State Functional Magnetic Resonance Imaging Data Processing. PLoS One 2011, 6(9):e25031.

45. Angeles Fernandez-Gil M, Palacios-Bote R, Leo-Barahona M, Mora-Encinas JP: Anatomy of the brainstem: a gaze into the stem of life. Semin Ultrasound CT MR 2010, 31(3):196-219.

46. Hurley RA, Flashman LA, Chow TW, Taber KH: The brainstem: anatomy, assessment, and clinical syndromes. J Neuropsychiatry Clin Neurosci 2010, 22(1):1-7. iv

47. Stoodley CJ, Valera EM, Schmahmann JD: Functional topography of the cerebellum for motor and cognitive tasks: an fMRI study. Neuroimage 2011, 59(2):1560-1570.

48. De Zeeuw Cl, Hoebeek FE, Bosman LW, Schonewille M, Witter L, Koekkoek SK: Spatiotemporal firing patterns in the cerebellum. Nat Rev Neurosci 2011, 12(6):327-344.

49. Kalenine S, Buxbaum LJ, Coslett HB: Critical brain regions for action recognition: lesion symptom mapping in left hemisphere stroke. Brain 2010, 133(11):3269-3280.

50. Kiriyama I, Miki H, Kikuchi K, Ohue S, Matsuda S, Mochizuki T: Topographic analysis of the inferior parietal lobule in high-resolution 3D MR imaging. AJNR Am J Neuroradiol 2009, 30(3):520-524.

51. Carter RM, O'Doherty JP, Seymour B, Koch C, Dolan RJ: Contingency awareness in human aversive conditioning involves the middle frontal gyrus. Neurolmage 2006, 29(3):1007-1012.

52. Van Rooij AJ, Schoenmakers TM, Vermulst AA, Van den Eijnden RJ, Van de Mheen D: Online video game addiction: identification of addicted adolescent gamers. Addiction 2010, 106(1):205-212.

53. Charlton JP, Danforth IDW: Distinguishing addiction and high engagement in the context of online game playing. Comput Hum Behav 2007, 23(3):1531-1548.

54. Wallace P: The psychology of the Internet. Cambridge: Cambridge University Press; 1999.

55. Robins DL, Hunyadi E, Schultz RT: Superior temporal activation in response to dynamic audio-visual emotional cues. Brain Cogn 2009, 69(2):269-278.

56. Lewald J, Staedtgen M, Sparing R, Meister IG: Processing of auditory motion in inferior parietal lobule: evidence from transcranial magnetic stimulation. Neuropsychologia 2011, 49(2):209-215.

57. Allison T, McCarthy G, Nobre A, Puce A, Belger A: Human extrastriate visual cortex and the perception of faces, words, numbers, and colors. Cereb Cortex 1994, 4(5):544-554.

58. Tam EW, Widjaja E, Blaser SI, Macgregor DL, Satodia P, Moore AM: Occipital lobe injury and cortical visual outcomes after neonatal hypoglycemia. Pediatrics 2008, 122(3):507-512.

59. Kojima H, Suzuki T: Hemodynamic change in occipital lobe during visual search: visual attention allocation measured with NIRS. Neuropsychologia 2010, 48(1):349-352. 
60. Battelli L, Alvarez GA, Carlson T, Pascual-Leone A: The role of the parietal lobe in visual extinction studied with transcranial magnetic stimulation. J Cogn Neurosci 2009, 21(10):1946-1955.

61. Yin TC: The parietal lobe and visual attention. J Psychiatr Res 1978, 14(1-4):261-266.

62. Yin TC, Mountcastle VB: Mechanisms of neural integration in the parietal lobe for visual attention. Fed Proc 1978, 37(9):2251-2257.

doi:10.1186/1744-9081-8-41

Cite this article as: Dong et al:: Alterations in regional homogeneity of resting-state brain activity in internet gaming addicts. Behavioral and Brain Functions 2012 8:41.

\section{Submit your next manuscript to BioMed Central and take full advantage of:}

- Convenient online submission

- Thorough peer review

- No space constraints or color figure charges

- Immediate publication on acceptance

- Inclusion in PubMed, CAS, Scopus and Google Scholar

- Research which is freely available for redistribution 